\title{
ARQUITECTURA NEURONAL PARA PREDICCIÓN DE RADIACIÓN SOLAR EN BASE A VARIABLES METEOROLÓGICAS
}

\section{NEURAL ARCHITECTURE FOR SOLAR RADIATION PREDICTION BASED ON METEOROLOGICAL VARIABLES}

\author{
Llinet Benavides*, Miguel Ángel Manso
} Departamento de Ingeniería Topográfica y Cartográfica, Escuela Técnica Superior de Ingenieros en Topografía, Geodesia y
Cartografía, Universidad Politécnica de Madrid, Campus Sur, A-3, Km 7, 28031 Madrid, España. Ilinet.bcesar@upm.es; m.manso@upm.es

\begin{abstract}
:
Solar forecasting is of great interest due to the growing use of renewable energies as an alternative to the global problems posed by current energy sources. In the last decade important advances have been achieved, thanks to the introduction of results from other areas of knowledge such as Artificial Intelligence. In particular, deep learning with the many variants of neural networks. In this paper, we propose a model that combines a convolutional layer with a GRU (Gated Recurrent Units) layer to a solution based on supervised classification. The classes were defined as intervals of solar radiation values. Objects are represented by ambient temperature, wind speed, atmospheric pressure, humidity and precipitation values. A dataset with thousands of records and a representativeness of two years is available, taken from meteorological stations distributed throughout Galicia. The evaluation was done using Macro-F1 measure because the classes are unbalanced.
\end{abstract}

Key words: supervised classification, recurrent neural networks, solar radiation

\section{Resumen:}

La predicción de la radiación solar presenta gran interés, debido al creciente uso de las energías renovables, como alternativa a los problemas globales que plantean las fuentes de energía actuales. En la última década se han logrado importantes avances, gracias a la introducción de resultados de otras áreas del conocimiento como la Inteligencia Artificial. En particular el aprendizaje profundo con las numerosas variantes de redes neuronales. Proponemos una solución basada en clasificación supervisada que combina en un modelo, una capa convolucional con una capa GRU (Gated Recurrent Units por sus siglas en inglés). Las clases se definen como intervalos de valores de radiación solar. Los objetos se representan a través de valores de temperatura ambiente, velocidad del viento, presión atmosférica y precipitación. Se dispone de un conjunto de datos de 112 estaciones con miles de registros de un período de dos años, tomados de estaciones meteorológicas distribuidas en todo el territorio de Galicia. Se ha empleado la métrica Macro-F1 para evaular la bondad de la clasificación porque las clases están desbalanceadas.

Palabras clave: clasificación supervisada, redes neuronales recurrentes, radiación solar

\section{Introducción}

Para el año 2030 el gobierno de España prevé a partir del Plan Nacional Integrado de Energía y Clima 2021-2030 (PNIEC), una generación eléctrica con potencia total instalada de $157 \mathrm{GW}$. De las cuales se espera que la solar fotovoltaica aporte 37 GW (BOE 2021). La predicción de radiación solar juega un papel muy importante, para mejorar la eficiencia de la entrega, de esta energía renovable de los productores a los consumidores. Al ser una fuente de energía que responde a los cambios en el clima y por tanto es variable, es importante estudiar cómo se comporta en base al histórico de medidas de las variables meteorológicas temperatura y presión atmosférica, entre otras. Este estudio se ha realizado con diferentes enfoques; modelos estadísticos ó modelos físicos, dentro de los métodos clásicos estadísticos de Aprendizaje Automatizado y variantes híbridas de estos métodos o con técnicas de Aprendizaje Profundo (Ahmad et al. 2020; Aslam et al. 2019; Shamshirband et al. 2019; Mellit et al. 2020); Nosotros nos centraremos en los que utilizan el aprendizaje profundo.

Uno de los retos actuales es definir arquitecturas de redes de aprendizaje profundo, capaces de predecir la radiación solar en base a las observaciones registradas por estaciones meteorológicas, entrenando las redes con un largo y extenso histórico de datos de los últimos años. Para aprender cómo afectan los cambios que se producen en la climatología (temperatura, presión atmosférica, velocidad del viento y dirección del mismo) a corto y medio plazo, evaluando cómo será el comportamiento de la radiación solar.

El enfoque de clasificación supervisada utilizado es novedoso en relación a lo investigado y descrito en la literatura. La mayoría de las aproximaciones se basan en predecir el valor de la radiación solar para un instante de tiempo o para un intervalo de tiempo, del modo más exacto posible. Nuestro objetivo, en este caso, es determinar si el nivel de radiación se encuentra dentro de un intervalo de radiaciones dado. Con tal propósito

“Corresponding Author: Llinet Benavides, llinet.bcesar@upm.es 
proponemos transformar el conjunto de datos, de tal forma que los valores de salida sean un vector de clases, en el que cada clase se corresponda con un intervalo de radiaciones. La propuesta consiste en clasificar o estimar en qué intervalo se encontrará la radiación solar usando el rango de 0 a $1300 \mathrm{wm}^{-2}$ a intervalos de 50 y $100 \mathrm{wm}^{-2}$.

Esta transformación de valores de radiación a un conjunto de clases, hace que el método de aprendizaje propuesto no se entrene para resolver un problema de regresión sino para uno de clasificación. La salida esperada del modelo es un vector que contenga las probabilidades de que el valor real que se desea predecir se encuentre en algunos de los intervalos seleccionados.

La parte restante del documento se organiza de la siguiente manera. Sección 1.1 que describe trabajos relacionados con la temática. Sección 2 en la que se describe la arquitectura empleada y el flujo de trabajo usado para obtener el resultado. La sección 3 es la presentación del conjunto de datos y el preprocesado realizado. Las secciones 4 y 5 muestran las medidas de evaluación y los experimentos, con un análisis crítico. Finaliza con la sección 6 con las conclusiones y el trabajo futuro.

\subsection{Trabajos relacionados}

Las técnicas de aprendizaje automatizado y en especial las de aprendizaje profundo dependen de un histórico de datos y predicen el comportamiento futuro sobre esa base. Para la predicción de radiación solar, el histórico de datos utilizado consta de variables observadas mediante estaciones meteorológicas desplegadas sobre un territorio o región (Carrera and Kim 2020; Kim et al. 2019), de imágenes de satélite (Babar et al. 2019; Ghimire et al. 2019), de imágenes del cielo (Pedro et al. 2019) (tomadas desde la superficie para analizar la claridad de la atmósfera) y modelos físicos (Yang et al. 2019). En las aproximaciones al problema se han usado las fuentes de datos de forma independiente o mezclando variables procedentes de las diferentes fuentes (Tao et al. 2021; Aguiar et al. 2016; Lotfi et al. 2020).

Recientemente en la predicción de radiación solar se han utilizado modelos de aprendizaje profundo que están establecidos en otros campos del conocimiento como el Procesamiento del Lenguaje Natural, la visión por computador y el tratamiento de imágenes médicas. En (Aslam et al. 2019) se realiza una revisión de los principales modelos usados, donde las redes recurrentes $y$ las redes convolucionales tienen una gran representación. Esto se debe a su capacidad de trabajar con secuencias de datos, aprovechando al máximo esa configuración de dataset de entrenamiento, para extraer características esenciales que contribuyen a mejorar la capacidad de predicción del modelo.

\section{Método propuesto}

El uso de redes neuronales convolucionales (CNN) es muy común en el campo del procesamiento de imágenes. Con su introducción, se logró la automatización del proceso de aplicación de filtros, obteniendo resultados excelentes para dicha tarea (filtrar). Pero no es el único campo que se ha visto beneficiado con su aplicación. En el campo del procesamiento de señales es muy común aplicar varias operaciones de convolución a la señal que se esté procesando con el objetivo de reducir ruido, suavizar la curva de la señal y extraer características significativas.

Las redes LSTM (Long-Short Term Memory por sus siglas en inglés) y GRU son redes neuronales recurrentes especialmente diseñadas para el análisis de secuencias de datos. Sin embargo, las redes GRU tienen una menor complejidad computacional que las LSTM, permitiendo completar los ciclos de entrenamiento en un menor tiempo. Por este motivo se escogió la red GRU para continuar con la investigación.

Sobre esta base se decidió implementar un modelo que combina una capa convolucional con una capa GRU. Con la estructura que se muestra en la Figura 1.

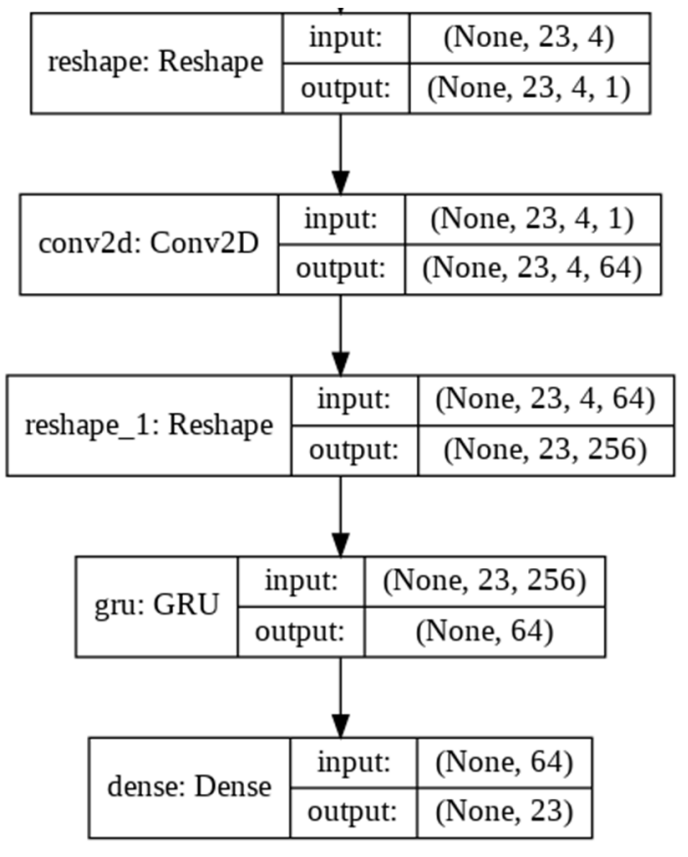

Figura 1: Arquitectura de la red.

La arquitectura comienza con la trasformación de los datos en la estructura de entrada que espera la capa convolucional, para procesarlos con una red convolucional con un filtro de $3^{*} 1$, se realiza una nueva transformación para la entrada de la capa GRU. La capa densa involucra un clasificador (o función de activación) softmax.

El modelo se entrena y valida a través de la iteración sobre el conjunto completo de datos. Usando un batchsize de 256 y num-epochs de 40 . La tasa de aprendizaje es de 0.0001 y el optimizador empleado es Adam. Los parámetros se obtuvieron a través de validación cruzada.

Se crearon 2 juegos de datos por estación como se muestra en la Figura 2, donde los valores de salida fueran un vector de clases, cada clase corresponde a un intervalo de radiaciones. En este caso en particular se seleccionaron 13 y 23 intervalos para un rango de 0 a $1300 \mathrm{wm}^{-2}$, los niveles de radiación posibles, con intervalos de 100 y de $50 \mathrm{wm}^{-2}$ respectivamente. 


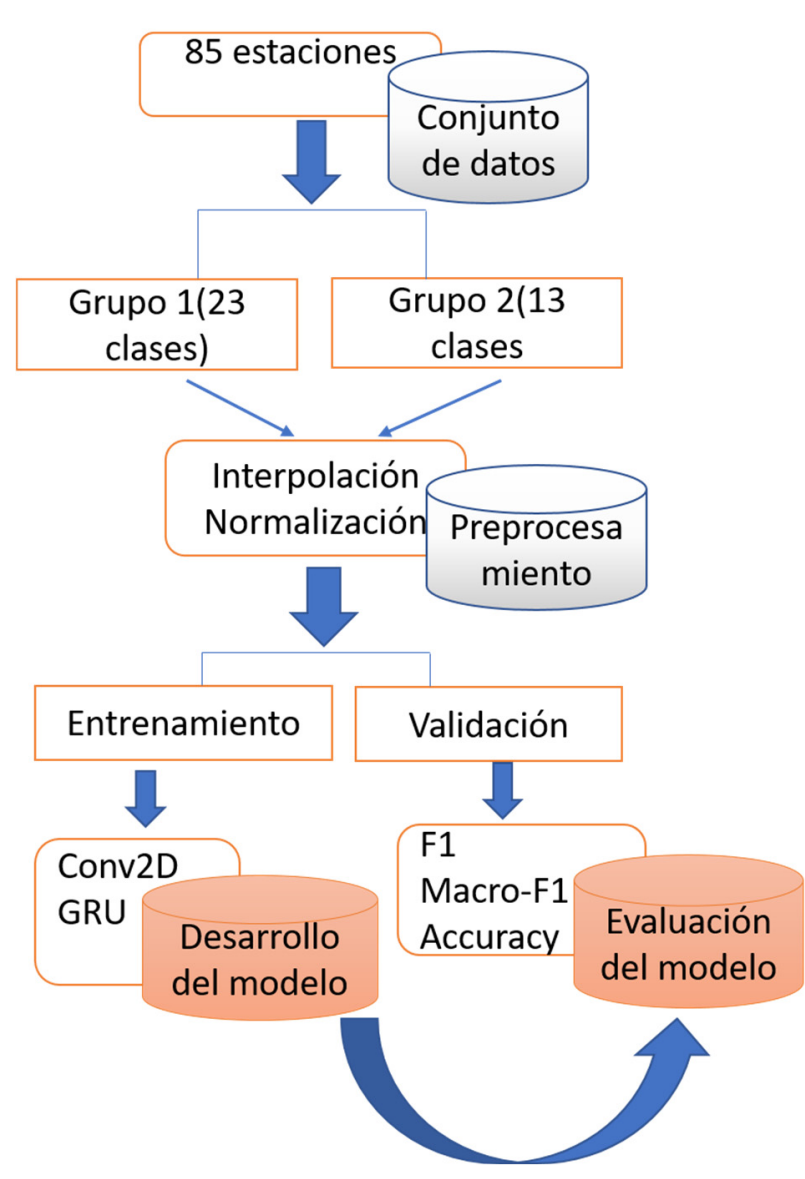

Figura 2: Flujo de trabajo.

Para cada uno de los juegos de datos se realizó un preprocesamiento de los datos, consistente en la normalización y una interpolación lineal. La normalización se estableció en un rango de $(-1,1)$.

\section{Descripción del conjunto de datos}

El conjunto de datos recopilado para la realización de esta investigación está compuesto por los registros de 112 estaciones meteorológicas de Galicia, almacenados en un archivo con formato tabular .csv. Las variables observadas fueron: la temperatura, la presión atmosférica, la precipitación, la velocidad y dirección del viento y la irradiancia solar. En la Figura 3 se muestran las locaciones de las estaciones utilizadas. Además se cuenta con un identificador para cada una de las estaciones, asi como sus coordenadas geográficas y su altura.

Sobre los datos se realizó una agrupación de los registros por estaciones, y se detectó que 27 estaciones no contenían información sobre la presión atmosférica. Este pre-procesamiento inicial provocó que el conjunto de datos contara con menos estaciones para el análisis. Los intervalos de tiempo de 10 minutos de cada una de las estaciones fueron utilizados para realizar la transformación de los datos y prepararlos para el entrenamiento de la red neuronal. Se utilizaron 85 estaciones para los experimentos. Con información de dos años, de febrero del 2017 a febrero del 2019.

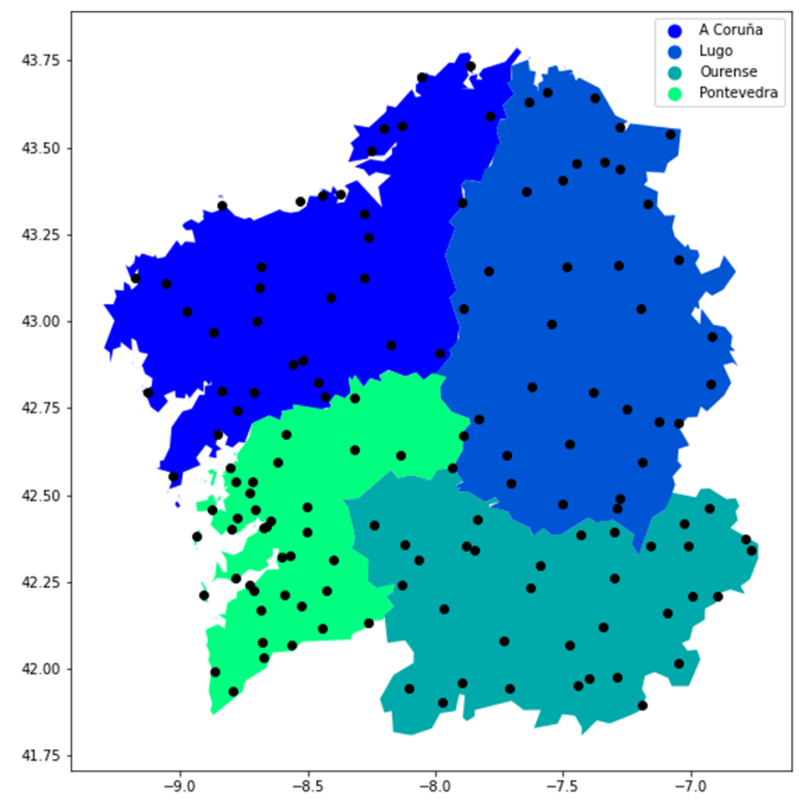

Figura 3: Representación espacial de las estaciones estudiadas.

\section{Métrica para la evaluación}

Para la evaluación de los resultados utilizamos la métrica Macro-F1 y la cantidad de aciertos de objetos clasificados correctamente, porque esta nos permite analizar la calidad de un problema de clasificación, en problemas con clases desbalanceadas Ec. (1):

Macro $-F 1=\frac{\sum F 1 i}{\mathrm{n}}$

F1 se calcula haciendo la media armónica entre la precisión (precision) y la exhaustividad (recall) para cada una de las clases Ec. (2):

$\mathrm{F} 1=2 * \frac{\text { Presicion } * \text { Recall }}{\text { Presicion }+ \text { Recall }}$

\section{Experimentos y análisis de los resultados}

Para la validación del modelo se dividió el conjunto de datos en $80 \%$ para el entrenamiento y $20 \%$ para la validación, para cada una de las estaciones en los grupos de datos y se evaluó utilizando las métricas mencionadas antes.

En la Figura 4 se muestra la evaluación del modelo por grupos de intervalos, Las líneas de color rojo representan los intervalos de $50 \mathrm{wm}^{-2}$ y las líneas de color azul representan los intervalos de $100 \mathrm{wm}^{-2}$.

Las líneas continuas representan los valores de aciertos y las líneas discontinuas los valores de Macro-F1 para cada una de las estaciones. Los valores de las medidas se muestran normalizadas en una escala de 0 a 1 , considerando el 1 como el $100 \%$ de calidad.

Como se puede apreciar en la Figura 4 para los valores de radiación agrupados en intervalos de $100 \mathrm{wm}^{-2}$ los niveles de acierto son más altos, entre un 0,6329 y 0,9107 , y la media obtiene un 0,7466 . No sucede lo mismo con los intervalos de $50 \mathrm{wm}^{-2}$ donde el acierto se encuentra entre 0,4660 y 0,8581 siendo la media un 
0,5969. Existen varios factores que afectan; el desbalance de clases, siendo las clases de los valores de radiación más pequeños, las de mayor representación, además que los límites de decisión entre clases están muy cercanos, creando confusión en la clasificación de los objetos.
La medida Macro-F1 para los intervalos de 100 alcanza valores entre 0,3940 y 0,6544 , con una media de 0,5127 . En el caso de los intervalos de 50 son menores los valores entre 0,2356 y 0,5352 , con una media de 0,3553 . Esta medida se afecta principalmente por los errores en la clasificación para valores altos de radiación.

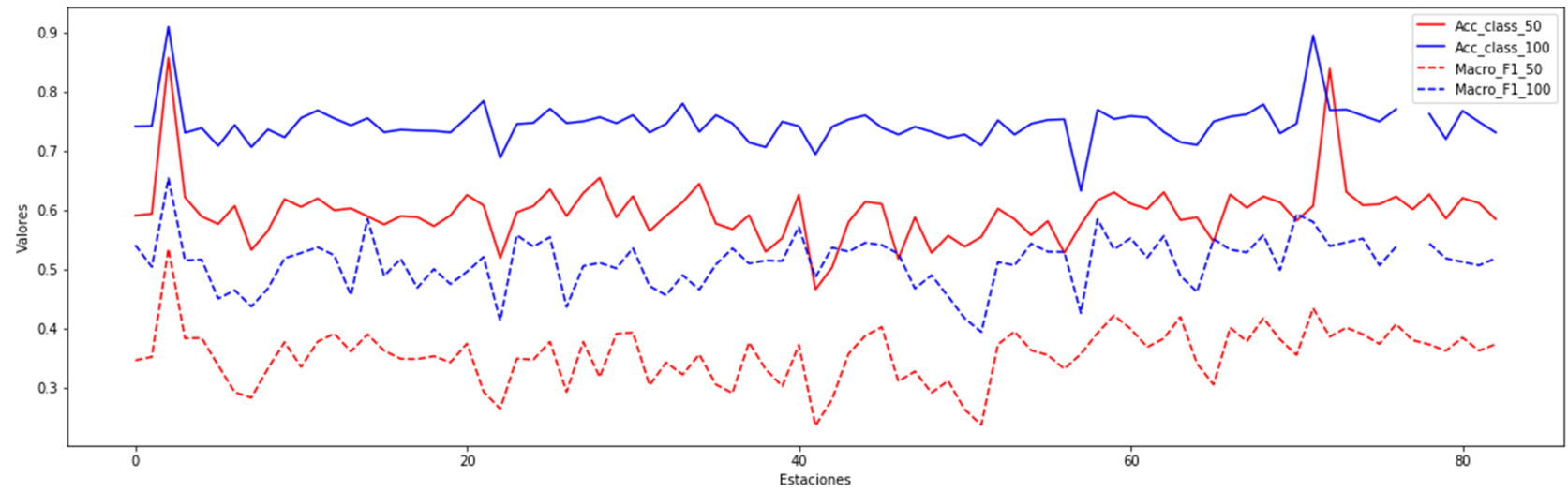

Figura 4: Comparación entre Aciertos y Macro-F1 en ambas tareas de clasificación.

\section{Conclusiones y Trabajo Futuro}

Se ha presentado un modelo de aprendizaje profundo para una tarea de clasificación supervisada, que combina una capa convolucional con una capa GRU. Los mejores resultados se han alcanzado para las clases de intervalos de $100 \mathrm{wm}^{-2}$, aunque se observó mayor complejidad en la clasificación en los intervalos de mayor radiación, debido fundamentalmente a la baja representatividad de estas clases. La métrica Macro-F1 se ve afectada principalmente por la clasificación para valores altos de radiación.
Proyectamos evaluar modelos de regresión con el mismo juego de datos empleando fundamentalmente aproximaciones recurrentes y modelos de regresión propuestos en el estado del arte. Ademas, considerar la localización de las estaciones mediante el uso de sus coordenadas y su altitud, para realizar análisis de proximidad o que la red aprenda a identificar las similitudes entre estaciones relativas a las localizaciones con el fin úlitmo de estudiar la predicción por vecinos cercanos.

\section{References}

AGUIAR, L. M., PEREIRA, B., LAURET, P., DÍAZ, F., and DAVID, M., 2016. Combining solar irradiance measurements, satellite-derived data and a numerical weather prediction model to improve intra-day solar forecasting. Renewable Energy, 97, pp. 599-610.

ASLAM, S., HERODOTOU, H., AYUB, N., and MOHSIN, S. M., 2019. Deep learning based techniques to enhance the performance of microgrids: a review. In 2019 International Conference on Frontiers of Information Technology (FIT), pp. 116-1165. IEEE. DOI: 10.1109/FIT47737.2019.00031.

AHMAD, T., ZHANG, H., and YAN, B., 2020. A review on renewable energy and electricity requirement forecasting models for smart grid and buildings. Sustainable Cities and Society, 55, 102052.

BABAR, B., LUPPINO, L. T., BOSTRÖM, T. and ANFINSEN, S. N., 2019. Random forest regression for improved mapping of solar irradiance at high latitudes. Solar Energy, 198, pp. 81-92.

CARRERA, B. and KIM, K., 2020. Comparison analysis of machine learning techniques for photovoltaic prediction using weather sensor data. Sensors (Switzerland), 20(11).

GHIMIRE, S., DEO, R. C., RAJ, N. and MI, J., 2019. Deep Learning Neural Networks Trained with MODIS Satellite-Derived Predictors for Long-Term Global Solar Radiation Prediction. Energies, 12(12).

KIM, S. G., JUNG, J. Y. and SIM, M. K., 2019. A two-step approach to solar power generation prediction based on weather data using machine learning. Sustain, 11(5).

LOTFI, M., JAVADI, M., OSÓRIO, G. J., MONTEIRO, C., and CATALÃO, J. P., 2020. A novel ensemble algorithm for solar power forecasting based on kernel density estimation. Energies, 13(1), 216.

MELLIT, A., MASSI PAVAN, A., OGLIARI, E., LEVA, S., and LUGHI, V., 2020. Advanced methods for photovoltaic output power forecasting: A review. Applied Sciences, 10(2), 487. 
BOE. 2021. BOE-A-2021-421 Resolución de 30 de diciembre de 2020, de la Dirección General de Calidad y Evaluación Ambiental, por la que se formula la declaración ambiental estratégica del Plan Nacional Integrado de Energía y Clima 2021-2030. Available: https://www.boe.es/diario_boe/txt.php?id=BOE-A-2021-421 [3/5, 2021].

PEDRO, H. T. C., COIMBRA, C. F. M. and LAURET, P., 2019. Adaptive image features for intra-hour solar forecasts. J. Renew. Sustain. Energy, 11(3), pp. 1-14.

SHAMSHIRBAND, S., RABCZUK, T. and CHAU, K.-W., 2019. A Survey of Deep Learning Techniques: Application in Wind and Solar Energy Resources. IEEE Access, 7, pp. 164650-164666.

TAO, H., EWEES, A. A., AL-SULtTANI, A. O., BEYAZTAS, U., HAMEED, M. M., SALIH, S. Q., ARMANUOS, A. M., ALANSARI, N., VOYANT, C., SHAHID, S., and YASEEN, Z. M., 2021. Global solar radiation prediction over North Dakota using air temperature: development of novel hybrid intelligence model. Energy Reports, 7, pp. 136-157.

YANG, D., WU, E. and KLEISSL, J., 2019. Operational solar forecasting for the real-time market. Int. J. Forecast, 35(4), pp. 1499-1519. 